\title{
Profil médical des patients en médecine bucco-dentaire à l'UCSA de la maison d'arrêt de Reims : étude rétrospective sur un an
}

\author{
Mathieu A, Furon V, Bonnet AL, Pierrard L, Lefèvre B. \\ (Pôle d'Odontologie, CHU de Reims)
}

Introduction :

La pratique de la médecine bucco-dentaire en milieu pénitentiaire est réalisée par le service public hospitalier dans les Unités de Consultation et de Soins Ambulatoires (UCSA). Les particularités de cette activité clinique sont liées aux contraintes de prise en charge inhérentes au milieu carcéral et à la population rencontrée, non représentative pour ses caractéristiques démographiques et médicales du reste de la population nationale.

\section{Objectif :}

Evaluer la prévalence des comorbidités associées chez les patients consultant en UCSA pour des soins buccodentaires.

\section{Méthodes :}

II s'agit d'une étude transversale monocentrique rétrospective sur l'ensemble des détenus ou prévenus soignés au cabinet dentaire de l'UCSA de la maison d'arrêt en 2009. Le recueil des données à partir des dossiers médicaux comprenait : les variables démographiques, les pathologies classées selon la Classification Internationale des Maladies de l'OMS (CIM-10), les statuts sérologiques et le suivi médical.

Résultats :

214 patients ont été inclus, l'âge moyen était de 29,5 \pm 10,4 ans (14-71 ans), 76,4\% des patients avaient moins de 35 ans. Tous les patients étaient de sexe masculin et 49,5\% avaient déjà fait l'objet d'une à sept incarcérations antérieures. 55 patients (25,7\%) étaient indemnes de pathologie autre que bucco-dentaire et $159(74,3 \%)$ avaient au moins une affection somatique et/ou psychiatrique décrite par la CIM-10 avec par ordre de fréquence : les troubles mentaux et du comportement $(59,8 \%)$, les maladies du système ostéo-articulaire, des muscles et du tissu conjonctif $(7,9 \%)$, les maladies de l'appareil circulatoire $(7 \%)$, les maladies infectieuses et parasitaires $(5,1 \%)$, les maladies respiratoires $(5,1 \%)$, les maladies du système nerveux $(4,2 \%)$, les autres groupes de maladies étaient chacun inférieurs à $3 \%$. Les statuts sérologiques révèlent 8 patients $\mathrm{VHC}+$ et un patient VHB+ et VHC+. La forte prévalence des troubles mentaux et du comportement était liée à la dépendance ou la consommation abusive de substances psychoactives : cannabis (22 cas) opiacés (34 cas) et surtout alcool (88 cas), pour 42 patients $(34,7 \%)$ il s'agissait de polyconsommation associant alcool et substances pyschoactives illicites. 117 patients $(54,9 \%)$ bénéficiaient d'un suivi psychologique et/ou psychiatrique dont 103 pour addiction.

\section{Discussion :}

Le profil démographique de cette série diffère de la population masculine nationale, mais est représentatif de la population carcérale avec une majorité de patients entre 20 et 39 ans (étude DREES 2005). Les troubles mentaux et du comportement y sont 4,5 fois plus élevés que dans la population générale masculine (étude IRDES 2010). La prévalence des maladies infectieuses chroniques, dominée par l'hépatite $C$, est proche de l'estimation nationale carcérale, mais inférieure aux chiffres des études internationales, où l'infection VIH et la tuberculose prédominent (Fasel et Baillargeon 2011). Les besoins médicaux en milieu pénitentiaire sont importants, les soins buccodentaires n'y dérogent pas et sont souvent réalisés dans un contexte d'urgence algique ou infectieuse en raison d'une santé bucco-dentaire précaire en relation avec les pathologies mentales, notamment addictives (Reece 2008). La nature et la prévalence des comorbidités constatées associées aux contraintes carcérales démontrent les spécificités de la médecine bucco-dentaire en UCSA.

MATHIEU Antoine

a-math@wanadoo.fr

This is an Open Access article distributed under the terms of the Creative Commons Attribution License 2.0, which permits unrestricted use, distribution, and reproduction in any medium, provided the original work is properly cited. 